\title{
Is a History of Falling Related to Oral Function? A Cross-Sectional Survey of Elderly Subjects in Rural Japan
}

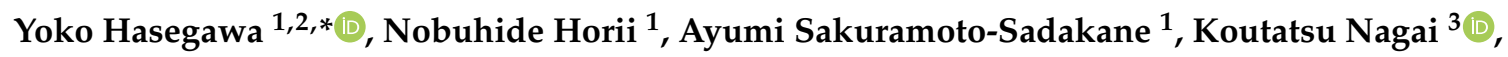 \\ Takahiro Ono ${ }^{2}$, Takashi Sawada ${ }^{4}$, Ken Shinmura ${ }^{5}$ and Hiromitsu Kishimoto ${ }^{1}$ \\ 1 Department of Dentistry and Oral Surgery, Hyogo College of Medicine, 1-1 Mukogawa-cho, Nishinomiya, \\ Hyogo 663-8501, Japan; no-horii@hyo-med.ac.jp (N.H.); ayu.cherry.ayu@gmail.com (A.S.-S.); \\ kisihiro@hyo-med.ac.jp (H.L.) \\ 2 Division of Comprehensive Prosthodontics, Graduate School of Medical and Dental Sciences, Niigata \\ University, Niigata 951-8514, Japan; ono@dent.niigata-u.ac.jp \\ 3 Department of Physical Therapy, School of Rehabilitation, Hyogo University of Health Sciences, 1-3-6 \\ Minatojima, Chuo-ku, Kobe, Hyogo 650-8530, Japan; nagai-k@huhs.ac.jp \\ 4 Hyogo Dental Association, 5-7-18 Yamamoto-dori, Chuo-ku, Kobe, Hyogo 650-0003, Japan; \\ ahfb4801@bca.bai.ne.jp \\ 5 Division of General Medicine, Department of Internal Medicine, Hyogo College of Medicine, 1-1 \\ Mukogawa-cho, Nishinomiya, Hyogo 663-8501, Japan; ke-shimmura@hyo-med.ac.jp \\ * Correspondence: cem17150@dent.niigata-u.ac.jp
}

Received: 19 September 2019; Accepted: 6 October 2019; Published: 11 October 2019

\begin{abstract}
Background: Deteriorated physical function makes older adults prone to fall, and it is therefore known to prompt elders to require long-term care. In this regard, oral function can be related to the loss of motor function. This cross-sectional study assessed the oral factors that increase the risk of falling among older adults. Methods: We surveyed 672 self-reliant elderly individuals aged $\geq 65$ years who were dwelling in a rural area. We assessed each subject's risk of falling and any related anxiety. Oral-related conditions (number of teeth, occlusal support, masticatory performance, occlusal force, and tongue pressure) and physical motor functions (gait speed, knee extension force, and one-legged standing) were also assessed. Statistical analyses were performed using Mann-Whitney's U-test, the $\chi^{2}$ test, and a logistic regression model. Results: In all subjects, $23 \%$ had a history of falling, while $40 \%$ had anxiety over falling. Both factors were significantly higher among female subjects, who also had slower gait speeds, and greater lateral differences in occlusion. The subjects with histories of falling were older, had impaired physical motor function, and exhibited a decrease in occlusal force and left/right occlusal imbalances. We recognized similar trends for anxiety about falling. Conclusions: These results revealed that the risk of falling might be lessened by maintaining healthy teeth occlusion and promoting healthy oral function.
\end{abstract}

Keywords: cross-sectional survey; elderly; falling; anxiety for falling; occlusal force; oral function; physical motor function

\section{Introduction}

Falls present one of the most serious and costly problems associated with older adulthood. Such incidents also strongly impact both health and quality of life for elderly people. In fact, approximately one in three people aged 65 and above experience at least one fall annually, and 10-15\% result in serious injury [1,2]. Accidents are the fifth leading cause of death among adults aged 65 years and older (after cardiovascular diseases, cancer, stroke, and respiratory causes) [3]. Falls account for two-thirds 
of all accidental deaths [4]. Decreased musculoskeletal strength in the limbs can make individuals more prone to falling. This is particularly known as a cause of falling among older adults who require caregiving. Falls are the main causes of accidental deaths among persons aged 65 years and older. Reports also indicate that older adults who fall once are at a higher risk of falling again. Fall prevention is thus important both socially and in terms of reducing medical costs.

Along with fall experience, excretory disorder, decreases in the frequency of going out, and depression, deteriorate oral function is now recognized as a risk related to caregiving needs among older adults [5]. The risk factors associated with falling include delayed reaction time, deteriorated skeletal muscle strength, decreased balance, and deteriorated gait function. This is evidence that declined physical function leads to an increase in fall frequency. Some reports suggest that skeletal muscle strength and oral function are related [6,7]. It is well known that when there is deterioration in physical equilibrium, this results in the decreased ability to perform activities of daily living (ADL) and the ability to stand without assistance (thereby increasing the risk of falling), and some reports also indicate that it is related to the loss of occlusal support $[8,9]$, which is thought to be related to the occurrence of falls. Improvement in physical equilibrium has also been reported as a result of improved masticatory function [10]. Furthermore, occlusal force is known to strongly influence both masticatory performance and food intake [11], while tooth loss decreases occlusal force and thereby reduces masticatory performance $[7,12]$. However, few studies have quantitatively examined and detailed the characteristics of oral function (i.e., occlusal force and masticatory performance) among older adults with recent falling experiences. Furthermore, while deteriorated physical motor function is associated with falls, there is insufficient evidence for the relationship between physical motor function and oral function in older adults with histories of falling.

This study revealed the relationship between oral function and the risk of falling, focusing on remaining teeth occlusion. In addition, we tried to determine the intra-oral factors that increase the risk of falling among older adults living in the Tanba-Sasayama area.

\section{Methods}

This cross-sectional study was approved by the institutional review board of Hyogo College of Medicine (approval no. Rinhi 0342) and is part of the Frail Elderly in the Tamba Sasayama-Area (FESTA) study. Tamba-Sasayama City is situated in the mountainous region of Hyogo prefecture. Its main industry is farming and agriculture. The city is aging more than by the average in Japan, and it is an area that seems to have a large distribution of elderly people (31.4\% were 65 or above as of September 2015). We recruited study participants by posting advertisements in local newspapers and by placing posters at the Hyogo Medical University Sasayama Medical Center.

\subsection{Research Subjects}

This study's subjects were 672 adults aged 65 and older (i.e., 223 males and 449 females aged $72.8 \pm 5.9$, mean \pm S.D.). These individuals participated in a joint medical and dental science study on independent older adults between June 2016 and December 2017. The participants were included based on the following criteria: participants were independent elderly individuals who required less than level 1 care based on the long-term care insurance system in Japan [13]. Exclusion criteria were participants suspected to have moderate to severe dementia (Mini-Mental State Examination score $<20$ ) [13], and those requiring level 2 or higher care based on the long-term care insurance system in Japan. If there was a suspicion of dementia in participants, their self-reported questionnaire was considered unreliable and they already often had problems with oral health [14]. Participants completed their own applications. Subjects were provided with full information about this study's purpose and methods and were required to provide written consent before participating.

The data used in this study were anonymized. All data were masked for analysis. The authors did not have access to any participants' personal information. 


\subsection{Evaluating the Risk of Falling}

A self-administered questionnaire survey was conducted using the Kihon checklist, which consists of 25 questions designed to screen individuals requiring care prevention in Japan [15]. We deemed subjects who responded "Yes" to "Have you fallen during the past year?" (hereinafter, history of falls) as those with a risk of falling. We also investigated subjects who responded "Yes" to "Do you have a lot of anxiety about falling?" (hereinafter, fall anxiety).

\subsection{Physical Component Assessments}

We evaluated bodily composition by performing bio-electrical impedance analyses with Inbody770 (Inbody Japan, Tokyo, Japan). We assessed skeletal muscle mass in the limbs according to the Skeletal Muscle Mass Index (SMI) [16]. Each subject's body mass index (BMI) was also calculated.

\subsection{Motor Function Evaluations}

We investigated the physical functions related to falling. Here, gait speed tests were performed by telling subjects to "walk at your normal speed". This was done to analyze normal gait speed $(\mathrm{m} / \mathrm{sec})$ (hereinafter, gait speed). Acceleration and deceleration were noted at the start and end of this process, respectively. Participants walked a total of $10 \mathrm{~m}$ but were given $1 \mathrm{~m}$ both before and after this measured range to accelerate and decelerate [17].

Knee extension force measurements were conducted using the manual muscle strength meter (Mobie, Sakai Medical Co., Ltd., Tokyo, Japan). Subjects were instructed to sit squarely on a bed with their knee joints bent at 90-degree angles (the buttocks did not rise). Measurements were taken twice with the dominant leg to assess maximum torque. Here, we used the value obtained after dividing torque by bodyweight [18].

Subjects were instructed to stand on one leg with their eyes open (hereinafter, standing on one leg). We evaluated the time (in seconds) starting from the moment the subject placed their hands on their waist and raised their dominant leg off the floor until the hands came off the waist, the position of the foot changed, or part of the body other than the supporting leg touched the floor [19]. This measurement was performed twice for each subject, with the fastest times being used for analysis.

\subsection{Oral Assessment}

Subjects were seated on a reclining care chair to receive oral examinations under bright artificial lighting. We obtained data on the number of remaining teeth, denture usage condition, occlusal force, occlusal support, masticatory performance, and tongue pressure. The number of remaining teeth included tooth stumps and third molars.

Maximum occlusal force was measured at the left and right first molars using the Occlusal Force-Meter GM10 (Nagano Keiki, Tokyo, Japan) [7]. Total occlusal force was thus evaluated. If a subject was missing their first molar tooth, measurements were taken with the closest tooth so that both upper and lower teeth were active in the biting process. Subjects wearing dentures for daily life had measurements taken while wearing them. The left/right side balance of the occlusal force (hereinafter, occlusal balance) was determined using the following formula:

Occlusal balance $(\%)=($ Difference between left/right occlusal forces/Total occlusal forces on the left and right side) $\times 100$

To assess masticatory performance, subjects were asked to chew gummy jelly 30 times before spitting it out. As such, masticatory performance was evaluated using a 10-point scale (i.e., $0-9=\min -\max$ ) [20]. Tongue pressure was measured using a balloon-probe type device (JMS tongue pressure meter, JMS, Hiroshima, Japan). Two maximum tongue pressure measurements were taken from each subject to determine mean values [21]. About the above two assessments, subjects wearing dentures for daily life had measurements taken while wearing them. 
Occlusal support was assessed according to the Eichner classification [22] (Group A: Occlusal support in all four occlusal supporting zones; Group B: Occlusal support in one to three occlusal supporting zones; and Group C: No occlusal support).

\subsection{Statistical Analysis}

After testing for the normality and homogeneity of variance, all data were submitted to the non-parametric method. The relationship between history of falling and fall anxiety as well as that between motor function and oral function were evaluated using either Mann-Whitney's U-test or the $\chi^{2}$ test. We then examined the relationship between physical function and oral function according to the Spearman correlation coefficient (i.e., the correlation between the statistically significant factors of history of falling and oral function). We performed logistic regression analyses (forced entry) using history of falling as the objective variable (Yes $=1$, No $=0)$ to elucidate the factors related to falling. Here, measurement items were determined according to Mann-Whitney's U-tests or $\chi^{2}$ tests $(p<0.1$ were explanatory variables, while age and sex were control variables). Analyses were performed for all subjects and also according to sex (significance levels were set at 5\%).

\section{Results}

Most participants were women (67\%). Furthermore, $23 \%$ of all subjects had experienced a fall during the past year, while $40 \%$ had anxiety about falling (Table 1). Subjects with one of either history or anxiety about falling accounted for 39\%, which indicated that falling was a familiar problem. Further, a history of falling and anxiety about falling were significantly more common among females.

Table 1. Subject characteristics.

\begin{tabular}{|c|c|c|c|c|}
\hline Variables & $\begin{array}{c}\text { Overall } \\
\text { (672 Subjects) }\end{array}$ & $\begin{array}{c}\text { Male } \\
\text { (223 Subjects) }\end{array}$ & $\begin{array}{c}\text { Female } \\
\text { (449 Subjects) }\end{array}$ & $p$-Value \\
\hline Age (years) & $72.8 \pm 5.9$ & $73.2 \pm 6.1$ & $72.6 \pm 5.8$ & 0.21 \\
\hline BMI & $22.5 \pm 2.9$ & $23.1 \pm 2.7$ & $22.2 \pm 2.9$ & 0.003 \\
\hline SMI & $6.4 \pm 0.9$ & $7.4 \pm 0.7$ & $6 \pm 0.6$ & $<0.001$ \\
\hline \multicolumn{5}{|l|}{ Questionnaire for falling } \\
\hline Has a history of falling & $151(22.5)$ & $38(17)$ & $113(25.2)$ & 0.02 \\
\hline Has anxiety about falling & $271(40.3)$ & $62(27.8)$ & $209(46.5)$ & $<0.001$ \\
\hline \multicolumn{5}{|l|}{$\begin{array}{l}\text { History of and anxiety } \\
\text { about falling }\end{array}$} \\
\hline $\begin{array}{l}\text { Has both history } \\
\text { and anxiety }\end{array}$ & $79(11.8)$ & $12(5.4)$ & $67(14.9)$ & $<0.001$ \\
\hline $\begin{array}{c}\text { Has one of either history } \\
\text { or anxiety }\end{array}$ & $264(39.3)$ & $76(34.1)$ & $188(41.9)$ & \\
\hline \multicolumn{5}{|l|}{ Motor function } \\
\hline Gait speed (m/s) & $1.5 \pm 0.2$ & $1.5 \pm 0.2$ & $1.5 \pm 0.2$ & 0.84 \\
\hline $\begin{array}{l}\text { Knee extensor force } \\
(\mathrm{N} \cdot \mathrm{m} / \mathrm{kg})\end{array}$ & $356.7 \pm 120.5$ & $459.5 \pm 118.5$ & $306 \pm 83.7$ & $p<0.001$ \\
\hline Standing on one leg (s) & $27.6 \pm 24.8$ & $29.7 \pm 27.1$ & $26.5 \pm 23.5$ & 0.18 \\
\hline \multicolumn{5}{|l|}{ Oral assesment } \\
\hline Number of teeth & $20 \pm 8.9$ & $19.6 \pm 9.4$ & $20.3 \pm 8.6$ & 0.83 \\
\hline Wearing denture & $318(47.3)$ & $111(49.8)$ & $207(46.1)$ & 0.21 \\
\hline Occlusal force (kgf) & $59 \pm 37$ & $66 \pm 42.6$ & $55.6 \pm 33.4$ & 0.01 \\
\hline Occlusal balance (\%) & $23.8 \pm 21.2$ & $26 \pm 22.5$ & $22.6 \pm 20.5$ & 0.06 \\
\hline $\begin{array}{l}\text { Mastication performance } \\
\text { (score) }\end{array}$ & $3.9 \pm 2.3$ & $4 \pm 2.4$ & $3.8 \pm 2.2$ & 0.32 \\
\hline $\begin{array}{l}\text { Tongue pressure }(\mathrm{kPa}) \\
\text { Occlusal support }\end{array}$ & $31.1 \pm 8.7$ & $31.7 \pm 9$ & $30.8 \pm 8.6$ & 0.16 \\
\hline
\end{tabular}


Table 1. Cont.

\begin{tabular}{ccccc}
\hline Variables & $\begin{array}{c}\text { Overall } \\
\text { (672 Subjects) }\end{array}$ & $\begin{array}{c}\text { Male } \\
\text { (223 Subjects) }\end{array}$ & $\begin{array}{c}\text { Female } \\
\text { (449 Subjects) }\end{array}$ & p-Value \\
\hline Group A & $292(43.5)$ & $93(41.7)$ & $199(44.3)$ & 0.23 \\
Group B & $268(39.9)$ & $85(38.1)$ & $183(40.8)$ & \\
Group C & $112(16.7)$ & $45(20.2)$ & $67(14.9)$ & \\
\hline
\end{tabular}

$p$-Value: Comparison between male and female. Mann-Whitney's U-test or $\chi^{2}$ test. SMI: Skeletal Muscle Mass Index Fall history: Subjects who responded "Yes" to "Have you fallen during the past year?". Fall anxiety: Subjects who responded "Yes" to "Do you have a lot of anxiety about falling?". Occlusal support: Eichner classification.

Aside from knee extension force and occlusal force, there were no differences between men and women in terms of motor function and oral function, respectively. The number of remaining teeth were almost identical to those presented by an investigation conducted by the Ministry of Health, Labour and Welfare [23].

Although a statistically significant correlation was observed between motor function and oral function (excluding occlusal balance), there was no strong correlation (Table 2). Occlusal force/masticatory performance showed a greater correlation coefficient with knee extension force compared to other items.

Table 2. Relationship between oral assessments and motor functions.

\begin{tabular}{|c|c|c|c|c|c|c|}
\hline \multirow{2}{*}{$\begin{array}{c}\text { Variables } \\
\text { Overall }\end{array}$} & \multicolumn{2}{|c|}{ Gait Speed } & \multicolumn{2}{|c|}{ Knee Extension Force } & \multicolumn{2}{|c|}{ Standing on One Leg } \\
\hline & $r$ & $p$-Value & $r$ & $p$-Value & $r$ & $p$-Value \\
\hline Number of teeth & $0.161^{* *}$ & $<0.001$ & $0.189^{* *}$ & $<0.001$ & $0.189 * *$ & $<0.001$ \\
\hline Occlusal force & $0.201 * *$ & $<0.001$ & $0.322 * *$ & $<0.001$ & $0.198 * *$ & $<0.001$ \\
\hline Occlusal balance & -0.002 & 0.968 & 0.018 & 0.646 & -0.061 & 0.117 \\
\hline Masticatory performance & $0.193^{* *}$ & $<0.001$ & $0.235^{* *}$ & $<0.001$ & $0.182 * *$ & $<0.001$ \\
\hline Tongue pressure & 0.07 & 0.07 & $0.216^{* *}$ & $<0.001$ & $0.149 * *$ & $<0.001$ \\
\hline Occlusal support & $-0.144^{* *}$ & $<0.001$ & $-0.172 * *$ & $<0.001$ & $-0.192 * *$ & $<0.001$ \\
\hline \multicolumn{7}{|l|}{ Male } \\
\hline Number of teeth & $0.162 *$ & 0.016 & $0.242 * *$ & $<0.001$ & $0.134 *$ & 0.047 \\
\hline Occlusal force & $0.237 * *$ & $<0.001$ & $0.284^{* *}$ & $<0.001$ & $0.188 * *$ & 0.005 \\
\hline Occlusal balance & -0.035 & 0.61 & 0.042 & 0.533 & 0.056 & 0.409 \\
\hline Masticatory performance & $0.248^{* *}$ & $<0.001$ & $0.272 * *$ & $<0.001$ & $0.187 * *$ & 0.006 \\
\hline Tongue pressure & 0.12 & 0.074 & $0.343^{* *}$ & $<0.001$ & $0.188^{* *}$ & 0.005 \\
\hline Occlusal support & -0.113 & 0.095 & $-0.252 * *$ & $<0.001$ & -0.119 & 0.08 \\
\hline \multicolumn{7}{|l|}{ Female } \\
\hline Number of teeth & $0.160 * *$ & 0.001 & $0.224^{* *}$ & $<0.001$ & $0.223^{* *}$ & $<0.001$ \\
\hline Occlusal force & $0.191 * *$ & $<0.001$ & $0.335^{* *}$ & $<0.001$ & $0.196 * *$ & $<0.001$ \\
\hline Occlusal balance & 0.01 & 0.828 & -0.048 & 0.313 & $-0.130 * *$ & 0.006 \\
\hline Masticatory performance & $0.167 * *$ & $<0.001$ & $0.259 * *$ & $<0.001$ & $0.179 * *$ & $<0.001$ \\
\hline Tongue pressure & 0.045 & 0.337 & $0.173^{* *}$ & $<0.001$ & $0.126^{* *}$ & 0.008 \\
\hline Occlusal support & $-0.162 * *$ & 0.001 & $-0.235^{* *}$ & $<0.001$ & $-0.237^{* *}$ & $<0.001$ \\
\hline
\end{tabular}

$r$ : Spearman correlation coefficient, ${ }^{* *}: p<0.001{ }^{*}: p<0.05$.

\subsection{Relationships between Falling Experience, Motor Function, and Oral Function}

Participants with histories of falling were clearly older, had deteriorated motor function, deteriorated occlusal force, and uneven left/right occlusal balance compared to those with no such histories (Table 3). 
Table 3. The relationships between falling experience, motor function, and oral function.

\begin{tabular}{|c|c|c|c|}
\hline Overall (672 Subjects) & Yes (151 Subjects) & No (521 Subjects) & $p$-Value \\
\hline Age (years) * & $73.6 \pm 6$ & $72.5 \pm 5.9$ & 0.04 \\
\hline BMI & $22.7 \pm 2.8$ & $22.5 \pm 2.9$ & 0.16 \\
\hline SMI & $6.4 \pm 0.9$ & $6.4 \pm 1$ & 0.54 \\
\hline \multicolumn{4}{|l|}{ Motor function } \\
\hline Gait speed $(\mathrm{m} / \mathrm{s})$ * & $1.4 \pm 0.2$ & $1.5 \pm 0.2$ & 0.009 \\
\hline Knee extension force $(\mathrm{N} \cdot \mathrm{m} / \mathrm{kg})$ * & $334.5 \pm 113.4$ & $363.1 \pm 121.8$ & 0.004 \\
\hline Standing on one leg $(\mathrm{s}) *$ & $23.2 \pm 21.6$ & $28.8 \pm 25.5$ & 0.006 \\
\hline \multicolumn{4}{|l|}{ Oral function test } \\
\hline Number of teeth & $18.9 \pm 9.3$ & $20.4 \pm 8.8$ & 0.06 \\
\hline Wearing denture & $79(52.3)$ & $239(45.9)$ & 0.096 \\
\hline Occlusal force $(\mathrm{kgf}) *$ & $53.1 \pm 34.9$ & $60.8 \pm 37.5$ & 0.03 \\
\hline Occlusal balance $(\%) *$ & $29 \pm 25.6$ & $22.2 \pm 19.5$ & 0.007 \\
\hline Masticatory performance (score) & $3.5 \pm 2.4$ & $4 \pm 2.2$ & 0.056 \\
\hline Tongue pressure $(\mathrm{kPa})$ & $30 \pm 8.9$ & $31.4 \pm 8.6$ & 0.08 \\
\hline \multicolumn{4}{|l|}{ Occlusal support } \\
\hline Group A & $55(36.4)$ & $237(45.5)$ & 0.14 \\
\hline Group B & $68(45)$ & $200(38.4)$ & \\
\hline Group C & $28(18.5)$ & $84(16.1)$ & \\
\hline Male (223 subjects) & Yes (38 subjects) & No (185 subjects) & $p$-Value \\
\hline Age (years) & $74.1 \pm 6.5$ & $73 \pm 6$ & 0.35 \\
\hline BMI & $22.8 \pm 2.9$ & $23.2 \pm 2.7$ & 0.44 \\
\hline SMI & $7.3 \pm 0.8$ & $7.4 \pm 0.7$ & 0.53 \\
\hline \multicolumn{4}{|l|}{ Motor function } \\
\hline Gait speed (m/s) & $1.4 \pm 0.2$ & $1.5 \pm 0.2$ & 0.07 \\
\hline Knee extension force $(\mathrm{N} \cdot \mathrm{m} / \mathrm{kg})$ & $440.1 \pm 129.7$ & $463.4 \pm 116.1$ & 0.11 \\
\hline Standing on one leg (s) & $27.8 \pm 23.5$ & $30.1 \pm 27.8$ & 0.63 \\
\hline \multicolumn{4}{|l|}{ Oral function test } \\
\hline Number of teeth * & $17.1 \pm 9.4$ & $20.1 \pm 9.3$ & 0.03 \\
\hline Wearing denture & $24(63.2)$ & $87(47.0)$ & 0.051 \\
\hline Occlusal force (kgf) & $57 \pm 37.8$ & $67.9 \pm 43.4$ & 0.16 \\
\hline Occlusal balance $(\%) *$ & $37.3 \pm 30.4$ & $23.7 \pm 19.8$ & 0.017 \\
\hline Masticatory performance (score) * & $3.3 \pm 2.3$ & $4.1 \pm 2.4$ & 0.04 \\
\hline Tongue pressure $(\mathrm{kPa})$ & $29.6 \pm 7.4$ & $32.1 \pm 9.2$ & 0.07 \\
\hline \multicolumn{4}{|l|}{ Occlusal support } \\
\hline Group A & $11(28.9)$ & $82(44.3)$ & 0.21 \\
\hline Group B & $18(47.4)$ & $67(36.2)$ & \\
\hline Group C & $9(23.7)$ & $36(19.5)$ & \\
\hline Female (449 subjects) & Yes (113 subjects) & No (336 subjects) & $p$-Value \\
\hline Age (years) * & $73.4 \pm 5.8$ & $72.3 \pm 5.8$ & 0.045 \\
\hline $\mathrm{BMI} *$ & $22.7 \pm 2.7$ & $22.1 \pm 3$ & 0.013 \\
\hline SMI & $6 \pm 0.6$ & $5.9 \pm 0.7$ & 0.18 \\
\hline \multicolumn{4}{|l|}{ Motor function } \\
\hline Gait speed $(\mathrm{m} / \mathrm{s})$ * & $1.4 \pm 0.2$ & $1.5 \pm 0.2$ & 0.047 \\
\hline Knee extension force $(\mathrm{N} \cdot \mathrm{m} / \mathrm{kg})$ & $300 \pm 82.7$ & $308.1 \pm 84$ & 0.21 \\
\hline Standing on one leg $(\mathrm{s}) *$ & $21.7 \pm 20.8$ & $28.2 \pm 24.1$ & 0.005 \\
\hline \multicolumn{4}{|l|}{ Oral function test } \\
\hline Number of teeth & $19.5 \pm 9.2$ & $20.5 \pm 8.4$ & 0.43 \\
\hline Wearing denture & $55(48.7)$ & $1527(45.20)$ & 0.30 \\
\hline Occlusal force (kgf) & $51.8 \pm 33.9$ & $56.9 \pm 33.2$ & 0.13 \\
\hline Occlusal balance $(\%)$ * & $26.3 \pm 23.3$ & $21.4 \pm 19.3$ & 0.04 \\
\hline Masticatory performance (score) & $3.6 \pm 2.4$ & $3.9 \pm 2.1$ & 0.39 \\
\hline
\end{tabular}


Table 3. Cont.

\begin{tabular}{cccc}
\hline Overall (672 Subjects) & Yes (151 Subjects) & No (521 Subjects) & $p$-Value \\
\hline Tongue pressure (kPa) & $30.2 \pm 9.3$ & $31 \pm 8.3$ & 0.41 \\
Occlusal support & & & \\
Group A & $44(38.9)$ & $155(46.1)$ & 0.41 \\
Group B & $50(44.2)$ & $133(39.6)$ & \\
Group C & $19(16.8)$ & $48(14.3)$ & \\
\hline
\end{tabular}

Variables are the same as in Table 1. Results are shown for all subjects. $p$-Value: Comparison between with/without fall history. Mann-Whitney's U-Test or $\chi^{2}$. *: There was a significant difference between those with and without fall history.

Males showed no difference in motor function between those with a history of falling and those without. However, those with a history of falling often had significantly decreased measurements for items related to oral function. In other words, subjects with a history of falling had fewer teeth, decreased masticatory performance, and (although not significant) exhibited low tongue pressure and swallowing power. The results for women were different; those with a history of falling had decreased motor function and poorer occlusal balance with respect to oral function compared to those without a history of falling. Thus, subjects with a history of falling had significantly slower gait speeds, less endurance when standing on one leg, and poorer occlusal balance.

\subsection{Relationships between Anxiety about Falling, Motor Function, and Oral Function}

Subject motor function and oral function were related to anxiety about falling with nearly the same trend seen for a history of falling (Table 4). That is, subjects with anxiety about falling exhibited significantly diminished motor and oral functions. Most male subjects with anxieties about falling had significantly lower motor and oral functions across all evaluated items when compared to subjects with no anxieties about falling. Women did not exhibit any significant differences among the items related to motor or oral function regardless of falling anxiety.

Table 4. The relationship between anxiety over falling, motor function, and oral function.

\begin{tabular}{|c|c|c|c|}
\hline Overall (672 Subjects) & Yes (271 Subjects) & No (401 Subjects) & $p$-Value \\
\hline Age (years) * & $73 \pm 6$ & $70 \pm 5.7$ & $<0.001$ \\
\hline BMI & $25.7 \pm 2.7$ & $21.6 \pm 3$ & 0.86 \\
\hline SMI * & $5.5 \pm 0.9$ & $5.9 \pm 1$ & $<0.001$ \\
\hline \multicolumn{4}{|l|}{ Motor function } \\
\hline Gait speed $(\mathrm{m} / \mathrm{s}) *$ & $1.4 \pm 0.3$ & $1.5 \pm 0.2$ & 0.029 \\
\hline Knee extension force $(\mathrm{N} \cdot \mathrm{m} / \mathrm{kg})$ * & $329.2 \pm 104$ & $375.3 \pm 127.3$ & $<0.001$ \\
\hline Standing on one leg $(\mathrm{s})^{*}$ & $25.4 \pm 24.5$ & $29 \pm 24.8$ & 0.017 \\
\hline \multicolumn{4}{|l|}{ Oral function test } \\
\hline Number of teeth * & $19.1 \pm 9.2$ & $20.7 \pm 8.6$ & 0.03 \\
\hline Wearing denture * & $176(52.2)$ & $141(43.9)$ & 0.02 \\
\hline Occlusal force $(\mathrm{kgf})$ * & $52.1 \pm 34$ & $63.7 \pm 38.2$ & $<0.001$ \\
\hline Occlusal balance (\%) & $24 \pm 23$ & $23.6 \pm 19.9$ & 0.48 \\
\hline Masticatory performance (score) * & $3.7 \pm 2.2$ & $4 \pm 2.3$ & 0.044 \\
\hline Tongue pressure $(\mathrm{kPa}) *$ & $29.8 \pm 8.6$ & $32 \pm 8.7$ & 0.003 \\
\hline \multicolumn{4}{|l|}{ Occlusal support } \\
\hline Group A & $109(40.2)$ & $183(45.6)$ & 0.096 \\
\hline Group B & $107(39.5)$ & $161(40.1)$ & \\
\hline Group C & $55(20.3)$ & $57(14.2)$ & \\
\hline
\end{tabular}


Table 4. Cont.

\begin{tabular}{|c|c|c|c|}
\hline Overall (672 Subjects) & Yes (271 Subjects) & No (401 Subjects) & $p$-Value \\
\hline Male (223 subjects) & Yes (62 subjects) & No (161 subjects) & $p$-Value \\
\hline Age (years) * & $75.2 \pm 6.4$ & $72.4 \pm 5.8$ & 0.002 \\
\hline BMI & $23 \pm 2.4$ & $23.1 \pm 2.8$ & 0.94 \\
\hline SMI & $7.4 \pm 0.6$ & $7.4 \pm 0.7$ & 0.89 \\
\hline \multicolumn{4}{|l|}{ Motor function } \\
\hline Gait speed $(\mathrm{m} / \mathrm{s}) *$ & $1.4 \pm 0.3$ & $1.5 \pm 0.2$ & 0.04 \\
\hline Knee extension force $(\mathrm{N} \cdot \mathrm{m} / \mathrm{kg})$ * & $431.1 \pm 103.1$ & $470.3 \pm 122.4$ & 0.03 \\
\hline Standing on one leg $(\mathrm{s}) *$ & $23.6 \pm 22.2$ & $32.1 \pm 28.5$ & 0.02 \\
\hline \multicolumn{4}{|l|}{ Oral function test } \\
\hline Number of teeth & $17.4 \pm 10.2$ & $20.5 \pm 9$ & 0.054 \\
\hline Wearing denture * & $37(59.7)$ & $74(46.0)$ & 0.046 \\
\hline Occlusal force $(\mathrm{kgf}) *$ & $50.9 \pm 36.7$ & $71.9 \pm 43.4$ & 0.001 \\
\hline Occlusal balance $(\%)$ & $30.6 \pm 27.3$ & $24.3 \pm 20.2$ & 0.31 \\
\hline Masticatory performance (score) * & $3.5 \pm 2.4$ & $4.2 \pm 2.4$ & 0.047 \\
\hline Tongue pressure $(\mathrm{kPa}) *$ & $29.1 \pm 9.1$ & $32.7 \pm 8.7$ & 0.009 \\
\hline \multicolumn{4}{|l|}{ Occlusal support* } \\
\hline Group A & $22(35.5)$ & $71(44.1)$ & 0.02 \\
\hline Group B & $20(32.3)$ & $65(40.4)$ & \\
\hline Group C & $20(32.3)$ & $25(15.5)$ & \\
\hline Female (449 subjects) & Yes (209 subjects) & No (240 subjects) & $p$-Value \\
\hline Age (years) * & $73.6 \pm 5.9$ & $71.7 \pm 5.6$ & $<0.001$ \\
\hline BMI & $22.4 \pm 2.8$ & $22.1 \pm 3$ & 0.32 \\
\hline SMI & $5.9 \pm 0.6$ & $6 \pm 0.7$ & 0.21 \\
\hline \multicolumn{4}{|l|}{ Motor function } \\
\hline Gait speed $(\mathrm{m} / \mathrm{s})$ & $1.5 \pm 0.3$ & $1.5 \pm 0.2$ & 0.18 \\
\hline Knee extension force $(\mathrm{N \eta m} / \mathrm{kg})$ & $299.3 \pm 83.4$ & $311.9 \pm 83.7$ & 0.13 \\
\hline Standing on one leg (s) & $26 \pm 25.2$ & $27 \pm 21.9$ & 0.24 \\
\hline \multicolumn{4}{|l|}{ Oral function test } \\
\hline Number of teeth & $19.7 \pm 8.8$ & $20.8 \pm 8.4$ & 0.15 \\
\hline Wearing denture & $104(50.0)$ & $102(42.5)$ & 0.07 \\
\hline Occlusal force (kgf) & $52.5 \pm 33.3$ & $58.3 \pm 33.3$ & 0.055 \\
\hline Occlusal balance (\%) & $22 \pm 21.3$ & $23.2 \pm 19.8$ & 0.3 \\
\hline Masticatory performance (score) & $3.7 \pm 2.1$ & $3.9 \pm 2.2$ & 0.36 \\
\hline Tongue pressure $(\mathrm{kPa})$ & $30 \pm 8.4$ & $31.4 \pm 8.7$ & 0.1 \\
\hline \multicolumn{4}{|l|}{ Occlusal support } \\
\hline Group A & $87(41.6)$ & $112(46.7)$ & 0.45 \\
\hline Group B & $87(41.6)$ & $96(40)$ & \\
\hline Group C & $35(16.7)$ & $32(13.3)$ & \\
\hline
\end{tabular}

Variables are the same as in Table 1. Results are shown for all subjects. $p$-Value: Comparison between with/without fall anxiety. Mann-Whitney's U-test or $\chi^{2}$. *: There was a significant difference between those with and without fall history.

\subsection{Factors Affecting History of Falling}

A logistic regression analysis revealed that sex, gait speed, and occlusal balance were significant explanatory variables affecting the history of falling among subjects (Table 5). Meanwhile the odds of occlusal balance were significant, but the confidence interval was 1.012 and the lower limit of the confidence interval is 1.003 , suggesting that the impact of occlusal balance on falling was weak. Here, being a woman, having a slower gait speed, and/or experiencing uneven left/right occlusal balance may be associated with an increased risk of falling. 
Table 5. Factors affecting fall history.

\begin{tabular}{|c|c|c|c|c|c|c|c|}
\hline \multicolumn{2}{|c|}{ History of Falls } & \multirow[t]{2}{*}{$B$} & \multirow[t]{2}{*}{ Wald } & \multirow[t]{2}{*}{$p$-Value } & \multirow{2}{*}{$\begin{array}{l}\text { Odds } \\
\text { Ratio }\end{array}$} & \multicolumn{2}{|c|}{ Confidence Interval } \\
\hline & & & & & & Lower & Upper \\
\hline \multirow[t]{12}{*}{ Overall } & Age & 0.005 & 0.058 & 0.810 & 1.005 & 0.967 & 1.043 \\
\hline & Sex* & 0.568 & 4.176 & 0.041 & 1.764 & 1.024 & 3.040 \\
\hline & Gait speed* & -0.910 & 4.581 & 0.032 & 0.403 & 0.175 & 0.926 \\
\hline & High knee extension & 0.000 & 0.021 & 0.885 & 1.000 & 0.998 & 1.002 \\
\hline & Standing on one leg & -0.007 & 2.037 & 0.153 & 0.993 & 0.984 & 1.002 \\
\hline & Number of teeth & 0.002 & 0.007 & 0.933 & 1.002 & 0.967 & 1.037 \\
\hline & Wearing denture & 0.063 & 0.054 & 0.816 & 1.065 & 0.629 & 1.804 \\
\hline & Occlusal force & 0.001 & 0.091 & 0.763 & 1.001 & 0.993 & 1.009 \\
\hline & Occlusal balance * & 0.012 & 7.275 & 0.007 & 1.012 & 1.003 & 1.022 \\
\hline & $\begin{array}{l}\text { Masticatory } \\
\text { performance }\end{array}$ & -0.026 & 0.165 & 0.685 & 0.975 & 0.862 & 1.103 \\
\hline & Tongue pressure & -0.009 & 0.549 & 0.459 & 0.991 & 0.969 & 1.014 \\
\hline & Constant & -0.627 & 0.105 & 0.746 & 0.534 & & \\
\hline \multirow[t]{8}{*}{ Male } & Age & -0.003 & 0.010 & 0.920 & 0.997 & 0.933 & 1.064 \\
\hline & Gait speed & -1.066 & 1.646 & 0.200 & 0.344 & 0.068 & 1.755 \\
\hline & Number of teeth & 0.008 & 0.057 & 0.812 & 1.008 & 0.946 & 1.074 \\
\hline & Wearing denture & 0.316 & 0.374 & 0.541 & 1.372 & 0.498 & 3.784 \\
\hline & Occlusal balance * & 0.019 & 5.639 & 0.018 & 1.019 & 1.003 & 1.035 \\
\hline & $\begin{array}{l}\text { Masticatory } \\
\text { performance }\end{array}$ & -0.037 & 0.109 & 0.742 & 0.964 & 0.773 & 1.202 \\
\hline & Tongue pressure & -0.015 & 0.437 & 0.509 & 0.985 & 0.943 & 1.029 \\
\hline & Constant & -0.090 & 0.001 & 0.978 & 0.914 & & \\
\hline \multirow[t]{4}{*}{ Female } & Age & 0.02 & 1.56 & 0.21 & 1.02 & 0.99 & 1.07 \\
\hline & BMI & 0.08 & 3.67 & 0.06 & 1.08 & 1.00 & 1.16 \\
\hline & Occlusal balance & 0.009 & 3.13 & 0.08 & 1.01 & 1.00 & 1.02 \\
\hline & Constant & -4.86 & 8.32 & 0.004 & 0.01 & & \\
\hline
\end{tabular}

History of fall was the objective variable (Yes $=1, \mathrm{No}=0$ ), variables in Table 3 which the result of Mann-Whitney's U-test or $\chi^{2}$ test were $p<0.1$ were explanatory variables (For occlusal support, Group A was a reference variables), and sex and age were control variables. *: Statistically significant explanatory variables.

A logistic regression analysis performed on participant sex indicated that occlusal balance was a significant explanatory variable for men. This suggests that balanced left/right occlusal force reduces the risk of falling. We did not identify any statistically significant explanatory variables among women.

\section{Discussion}

Epidemiologic studies have reported on the risk factors related to falling. The global annual rate of falling among individuals aged 65 is between $28 \%$ and $35 \%$ [2], and elderly falls are a trigger for nursing care. The survey on falls for elderly people revealed the factors of falls, and a prompt response to preventable factors among these is important for maintaining the functioning of the elderly. It is also important for reducing social health care costs. The causes of falls are divided into internal and external (e.g., slight steps and slippery floor) factors. Internal factors are known to be due to age-related changes such as skeletal muscle strength, visual loss, loss of balance, and changes in the higher nervous system and sensory system. In the report of risk factors for falls, skeletal muscle weakness was the highest risk factor for fall risk [24]. Reduced skeletal muscle mass is known to be associated with decreased nutrition intake. In this cross-sectional survey, we inspected the hypothesis that decreased oral function is associated with decreased nutritional intake and decreased skeletal muscle mass and physical activity, resulting in an increased risk of falls.

This survey evaluated not only the incidence of falls but also anxiety about falls, because the reports on anxiety about falling have also indicated that it is a risk factor for falling [25].

Our findings showed that a decrease in gait speed increases the risk of falling. Decreased gait speed has often been considered the strongest risk factor for adverse events like falling among older adults; it is also one of the most useful indicators for determining physical frailty [26,27]. It is also 
true that decreased lower-limb muscle strength and less endurance when standing on one leg are risk factors for falling $[28,29]$.

This study's results suggest a correlation between oral function and history of falling. In addition, older adults with poor occlusal balance are at high risk of falling, while elderly men with fewer teeth and lower masticatory performance tend to have more frequent histories of falling and greater associated fear. Furthermore, while there was no clear correlation between history/fear of falling and oral function (other than occlusal balance) among female subjects, they were generally at a greater risk of falling than male subjects. Women report more falls and experience more fall-related injuries than men $[25,30]$. While women tend to have more gait variability (variation in steps) and are therefore more likely to fall than men [31], elderly women have the additional risk of advanced post-menopausal osteoporosis and fractures, suffering during falls, and decreased ADL and quality of life. Particular efforts are thus needed to prevent falls among these individuals.

This study's authors previously reported a correlation between occlusal balance and standing on one leg [32,33]. Kimura et al. further reported that time standing on one leg can be prolonged through dental treatment [34], while Ringhof et al. showed that occlusal control can stabilize normal posture and balance when standing on one leg [35]. Kimura et al. also reported that occlusal support while wearing dentures was related to Timed Up and Go and standing on one leg balance test results [34]. Thus, occlusal balance affects the balance of the body trunk in addition to lower-limb muscle strength and balance. Improvements to these areas therefore reduce the risk of falling. For this reason, it is necessary to restore oral function among older adults with significant left/right occlusal force imbalances.

This study was a cross-sectional survey and did not clarify a causal relationship with falling. The survey population mainly consisted of women. In addition, while occlusal force was assessed on the left and right first molars or neighboring teeth of each participant, occlusal force balance was not evaluated in a strict sense. Fall history in this study was confirmed through self-reports during the past year, so that self-reports have limited accuracy or reliability.

\section{Conclusions}

This study's results suggest that it is important to prevent falling accidents not only by slowing the deterioration of motor function, but also by promoting and maintaining healthy masticatory performance and occlusal force. Dental treatment may also be beneficial for at-risk individuals with poor left/right occlusal balance. Furthermore, while anxiety about falling was associated with decreased exercise, it was also significantly associated with deteriorated oral function (e.g., occlusal force, tongue pressure, and masticatory performance). Thus, efforts to improve oral function among older adults may also prevent falling and remove falling-related anxieties.

Author Contributions: Conceptualization, K.S. and H.K.; methodology, Y.H.; formal analysis, N.H. and A.S.-S.; investigation, Y.H., N.H.; writing—original draft preparation, Y.H., N.H. and K.N.; writing—review and editing, T.O., T.S. and K.S.; project administration, K.S.; funding acquisition, Y.H., T.S. and K.S.

Funding: This study was supported by the Foundation for Total Health Promotion, Hyogo Dental Association, Longevity Sciences (28-13) from the National Center for Geriatrics and Gerontology, Osaka Gas Group Welfare Foundation, 8020 foundations to Hasegawa Y and Kishimoto H, JSPS KAKENHI (Grant Number 16KT0012), and a grant given to Shinmura $\mathrm{K}$ to support collaborative investigation between the Hyogo College of Medicine and Hyogo University of Health Sciences.

Acknowledgments: The authors would like to express their sincere appreciation to M. Shiramizu for her tremendous support. The FESTA study team included Hiroo Yoshikawa, Sohji Shimomura, Akira Okayama, Yoshinori Ohta, Hiroshi Kusunoki, Yosuke Wada, Shotaro Tsuji, Mayuka Fukai, Kayoko Tamaki, Hatsuo Maeda, Manabu Amano, Masako Itoh, Kyoko Sano, Koutatsu Nagai, Shinichi Hirayama, Yukio Nogaki, Saki Shimizu, Masahiro Murakami, Megumi Yasuda, Shigefumi Murakami, Yoshiko Hatanaka, Atsushi Kondo, Beni Kira, Miyuu Ueno, Koji Yasuhira, Yukio Nogaki, Akane Inoue, and Masumi Nakanishi.

Conflicts of Interest: The authors declare no conflicts of interest. The founding sponsors had no role in the design of the study; in the collection, analyses, or interpretation of data; in the writing of the manuscript, or in the decision to publish the results. 


\section{References}

1. Sturnieks, D.L.; St George, R.; Lord, S.R. Balance disorders in the elderly. Neurophysiol Clin. 2008, 38, 467-478. [CrossRef] [PubMed]

2. Blake, A.J.; Morgan, K.; Bendall, M.J.; Dallosso, H.; Ebrahim, S.B.; Arie, T.H.; Fentem, P.H.; Bassey, E.J. Falls by elderly people at home: Prevalence and associated factors. Age Ageing 1988, 17, 365-372. [CrossRef] [PubMed]

3. Fuller, G.F. Falls in the elderly. Am. Fam. Physician 2000, 61, 2159-2168. [PubMed]

4. Paciaroni, M.; Mazzotta, G.; Corea, F.; Caso, V.; Venti, M.; Milia, P.; Silvestrelli, G.; Palmerini, F.; Parnetti, L.; Gallai, V. Dysphagia following stroke. Eur. Neurol. 2004, 51, 162-167. [CrossRef] [PubMed]

5. Hirai, K.; Kondo, H.; Ojima, T.; Murata, T. Investigation of risk factors for accreditation of need for care for community-dwelling elderly people: AGES project 3-year follow-up research. Jpn. J. Public Health 2009, 56, 501-512. [CrossRef]

6. Hämäläinen, P.; Rantanen, T.; Keskinen, M.; Meurman, J.H. Oral health status and change in handgrip strength over a 5-year period in 80-year-old people. Gerodontology 2004, 21, 155-160. [CrossRef] [PubMed]

7. Iinuma, T.; Arai, Y.; Fukumoto, M.; Takayama, M.; Abe, Y.; Asakura, K.; Nishiwaki, Y.; Takebayashi, T.; Iwase, T.; Komiyama, K.; et al. Maximum occlusal force and physical performance in the oldest old: The Tokyo oldest old survey on total health. J. Am. Geriatr. Soc. 2012, 60. [CrossRef] [PubMed]

8. Morikawa, E. Epidemiological study on the influence of maintaining and restoring occlusion on the balance of the body in the elderly. J. Hiroshima. Univ. Dent. 2007, 39, 24-36.

9. Nagai, H. Influence of masticatory performance on health condition in community-dwelling elderly. Jpn. J. Geriat. 1990, 27, 63-68.

10. Hagiwara, J.; Moriya, S.; Murata, A.; Harada, E.; Kobayashi, K.; Nodani, K.; Kashiwazaki, H.; Kuroe, T.; Inoue, $\mathrm{N}$. Influence of treatment of dental prostheses on body balance function in case of loss of occlusal support of posterior teeth. Hokkaido. J. Dent. Sci. 2009, 30, 80-86.

11. Kim, B.I.; Jeong, S.H.; Chung, K.H.; Cho, Y.K.; Kwon, H.K.; Choi, C.H. Subjective food intake ability in relation to maximal bite force among Korean adults. J. Oral Rehabil. 2009, 36, 168-175. [CrossRef] [PubMed]

12. Ikebe, K.; Matsuda, K.; Kagawa, R.; Enoki, K.; Yoshida, M.; Maeda, Y.; Nokubi, T. Association of masticatory performance with age, gender, number of teeth, occlusal force and salivary flow in Japanese older adults: Is ageing a risk factor for masticatory dysfunction? Arch. Oral Biol. 2011, 56, 991-996. [CrossRef] [PubMed]

13. Perneczky, R.; Wagenpfeil, S.; Komossa, K.; Grimmer, T.; Diehl, J.; Kurz, A. Mapping scores onto stages: Mini-mental state examination and clinical dementia rating. Am. J. Geriatr. Psychiatry 2006, 14, 139-144. [CrossRef] [PubMed]

14. Delwel, S.; Binnekade, T.T.; Perez, R.; Hertogh, C.; Scherder, E.J.A.; Lobbezoo, F. Oral hygiene and oral health in older people with dementia: A comprehensive review with focus on oral soft tissues. Clin. Oral Investig. 2018, 22, 93-108. [CrossRef]

15. Satake, S.; Senda, K.; Hong, Y.J.; Miura, H.; Endo, H.; Sakurai, T.; Kondo, I.; Toba, K. Validity of the Kihon Checklist for assessing frailty status. Geriatr. Gerontol. Int. 2016, 16, 709-715. [CrossRef] [PubMed]

16. Srikanthan, P.; Karlamangla, A.S. Relative Muscle Mass Is Inversely Associated with Insulin Resistance and Prediabetes. Findings from The Third National Health and Nutrition Examination Survey. J. Clin. Endocrinol. Metab. 2011, 96, 2898-2903. [CrossRef] [PubMed]

17. Lopopolo, R.B.; Greco, M.; Sullivan, D.; Craik, R.L.; Mangione, K.K. Effect of therapeutic exercise on gait speed in community-dwelling elderly people: A meta-analysis. Phys. Ther. 2006, 86, 520-540.

18. Wang, C.Y.; Olson, S.L.; Protas, E.J. Test-retest strength reliability: Hand-held dynamometry in community-dwelling elderly fallers. Arch. Phys. Med. Rehabil. 2002, 83, 811-815. [CrossRef]

19. MEXT. New Physical Fitness Test for Meaningful Use; MEXT, Ed.; Springer International Publishing: Gyosei, Japen, 2000.

20. Nokubi, T.; Yoshimuta, Y.; Nokubi, F.; Yasui, S.; Kusunoki, C.; Ono, T.; Maeda, Y.; Yokota, K. Validity and reliability of a visual scoring method for masticatory ability using test gummy jelly. Gerodontology 2013, 30, 76-82. [CrossRef]

21. Utanohara, Y.; Hayashi, R.; Yoshikawa, M.; Yoshida, M.; Tsuga, K.; Akagawa, Y. Standard values of maximum tongue pressure taken using newly developed disposable tongue pressure measurement device. Dysphagia 2008, 23, 286-290. [CrossRef] 
22. Eichner, K. Renewed examination of the group classification of partially edentulous arches by Eichner and application advices for studies on morbidity statistics. Stomatologie der DDR 1990, 40, 321-325. [PubMed]

23. MHLW. 2016 Survey on Dental Diseases. Available online: http://www.mhlw.go.jp/toukei/list/dl/62-28-01.pdf (accessed on 18 September 2019).

24. Guideline for the prevention of falls in older persons. American Geriatrics Society, British Geriatrics Society, and American Academy of Orthopaedic Surgeons Panel on Falls Prevention. J. Am. Geriatr. Soc. 2001, 49, 664-672.

25. Deandrea, S.; Lucenteforte, E.; Bravi, F.; Foschi, R.; La Vecchia, C.; Negri, E. Risk factors for falls in community-dwelling older people: A systematic review and meta-analysis. Epidemiology 2010, 21, 658-668. [CrossRef] [PubMed]

26. Gill, T.M.; McGloin, J.M.; Gahbauer, E.A.; Shepard, D.M.; Bianco, L.M. Two recruitment strategies for a clinical trial of physically frail community-living older persons. J. Am. Geriatr. Soc. 2001, 49, 1039-1045. [CrossRef] [PubMed]

27. Rothman, M.D.; Leo-Summers, L.; Gill, T.M. Prognostic significance of potential frailty criteria. J. Am. Geriatr. Soc. 2008, 56, 2211-2216. [CrossRef] [PubMed]

28. Horak, F.B.; Shupert, C.L.; Mirka, A. Components of postural dyscontrol in the elderly: A review. Neurobiol. Aging 1989, 10, 727-738. [CrossRef]

29. Vellas, B.J.; Wayne, S.J.; Romero, L.; Baumgartner, R.N.; Rubenstein, L.Z.; Garry, P.J. One-Leg Balance Is an Important Predictor of Injurious Falls in Older Persons. J. Am. Geriatr. Soc. 1997, 45, 735-738. [CrossRef]

30. Stevens, J.A.; Mack, K.A.; Paulozzi, L.J.; Ballesteros, M.F. Self-reported falls and fall-related injuries among persons aged $>$ or $=65$ years-United States, 2006. J. Safety Res. 2008, 39, 345-349. [CrossRef] [PubMed]

31. Johansson, J.; Nordstrom, A.; Nordstrom, P. Greater Fall Risk in Elderly Women Than in Men Is Associated With Increased Gait Variability During Multitasking. J. Am. Med. Director. Assoc. 2016, 17, 535-540. [CrossRef]

32. Hasegawa, Y.; Sakuramoto, A.; Sugita, H.; Hasegawa, K.; Horii, N.; Sawada, T.; Shinmura, K.; Kishimoto, H. Relationship between oral environment and frailty among older adults dwelling in a rural Japanese community: A cross-sectional observational study. BMC Oral Health 2019, 19, 23. [CrossRef]

33. Hasegawa, Y.; Horii, N.; Sakuramoto, A.; Sugita, H.; Ono, T.; Sawada, T.; Nagai, H.; Shinmura, K.; Kishimoto, H. Relationship between Accidental Fall Risk and Oral Function in Elderly Living in the Tamba Area. Ronen Shika Igaku 2018, 32, 468-476. [CrossRef]

34. Kimura, M.; Watanabe, M.; Tanimoto, Y.; Kusabiraki, T.; Komiyama, M.; Hayashida, I.; Kono, K. Occlusal support including that from artificial teeth as an indicator for health promotion among community-dwelling elderly in Japan. Geriatr. Gerontol. Int. 2013, 13, 539-546. [CrossRef] [PubMed]

35. Ringhof, S.; Stein, T.; Potthast, W.; Schindler, H.J.; Hellmann, D. Force-controlled biting alters postural control in bipedal and unipedal stance. J. Oral Rehabil. 2015, 42, 173-184. [CrossRef] [PubMed] 